\title{
New SCRAM Experimental Device at Training reactor VR-1
}

\author{
Ondrej Novak ${ }^{1}$ and Lubomir Sklenka ${ }^{1}$ \\ ${ }^{1}$ Czech Technical University in Prague \\ Faculty of Nuclear Sciences and Physical Engineering \\ Department of Nuclear Reactors \\ Prague, Czech Republic \\ V Holesovickach 2, 18000 Prague 8, Czech Republic \\ ondrej.novak2@fjfi.cvut.cz, lubomir.sklenka@fjfi.cvut.cz
}

\begin{abstract}
The paper deals with design of new SCRAM experimental device at Training reactor VR-1. Crucial works cowers the detector development, transient calculations and principal design of the device. Conceptual design will be prepared in 2019-2020. This paper presents current work status.
\end{abstract}

KEYWORDS: Reactor transients; SCRAM experiments ; instrumentation development; VR-1 reactor

\section{Introduction}

One of the crucial aspects of nuclear power plant operation is the immediate and safe stop of the nuclear chain reaction if needed, i.e. SCRAM. Clear understanding of the SCRAM mechanism, i.e. control rod drop to the core, and consequent evolution of neutron flux, is still important even though nuclear power plants have been in commercial operation for five decades. Theoretical calculations and computer modelling of reactor operational states, including transients, should be verified by experiments. Research reactors are suitable nuclear installations for carrying out verification experiments for many operational states of nuclear power plants. Standard research reactor technology and its experimental instrumentation usually cannot provide on-line information of control rod position during SCRAM. On-line data on neutron flux deformation during SCRAM are also usually not available. The typical SCRAM time at a research reactor is ca. 1-2 s (due to low pressure, temperature and coolant flow) distance between top and bottom position of control rod is ca 50-100 cm. This is why particular experimental control rods with on-line measurement of rod position during SCRAM should be developed as well as a new generation of neutron detectors (small, very sensitive with fast response and at a reasonable price).

The Training reactor VR-1 which is operated by Czech Technical University in Prague (CTU), Czech Republic is a well-established reactor for nuclear education and training as well as for research in various reactor transients. A new project at the VR-1 reactor was launched in 2017: development of new SCRAM experimental device which can be used for verification of theoretical calculations and computer modeling of SCRAMs at nuclear power plants and research reactors. International collaboration between the Czech Republic (CTU), France (CEA Saclay) and the USA 
(The University of Tennessee, Knoxville - UTK) will accumulate enough knowledge and experiences for the project to be finished in ca 4-5 years, i.e. in 2021-2022.

Since 2017, the diamond-based detectors are being developed in collaboration with CEA Saclay and now are in testing phase at the VR-1 reactor. Diamond detectors are rarely used in reactor physics experiments but are promising for this particular application. Conceptual design of the SCRAM device will be prepared in 2019-2020 using experiences from detectors developments and from a set of SCRAM experiments carried out at the VR-1 reactor. Detailed design is currently under discussion with a potential manufacturer as well as fundraising for the new device. It is expected that manufacturing, testing, and licensing the SCRAM device will take 1 year.

Along with the SCRAM device, potential utilization is also under development. In 2018-2019 theoretical calculations and computer modeling of SCRAMs is being carried out in collaboration with UTK. The Serpent code (as pilot model code) is used for precise simulations of reactor transient. The time-dependent transient calculation is based on the full-core 3D model of the VR-1 training reactor. The paper also presents some results from SCRAM experiments carried out at the VR-1 reactor which are used for the conceptual design and detail of the SCRAM experimental device.

\section{Training reactor VR-1}

The Training reactor VR-1 [1] [2] operated by Czech Technical University in Prague is a pool-type light-water reactor based on low-enriched (19.7\% of U-235) uranium fuel with a maximal thermal power $100 \mathrm{~W}$. A maximal thermal neutron flux is approximately $10^{11} \mathrm{n} / \mathrm{cm}^{2} \cdot \mathrm{s}^{-1}$. The neutron moderator is light demineralized water, which also serves as a reflector, a biological shield, and a coolant. Heat generated during operation is removed from the core by natural convection. The reactor consists of two cylindrical stainless-steel vessels-opened pools. The reactor core is housed in the first one. The second vessel is used for the temporary storage of fuel assemblies during fuel handling and the disassembly. The pools are separated from each other by a removable gate. The reactor schematics is shown in Figure 1.

The reactor core usually contains from 16 to 21 fuel assemblies IRT-4M, depending on the geometric arrangement and kind of experiments performed in the reactor. Regarding the core configuration, the VR-1 reactor is very flexible having a core assembly with several variations. The cadmium control rods are used for reactor control and safe shutdown. The reactor core contains from five to seven control rods depending on the core configuration. Construction of all the rods is identical, but they differ in their functions (safety, compensation, or control), according to the connection with the control and safety system. The digital control equipment consists of a control and safety system, signaling system, connecting system, and neutron source control. The reactor power is measured by four wide range fission chambers which in pulse or current modes depending on reactor power. The reactor is equipped with several dry vertical channels that allows for additional detector placement, radial, and tangential channels used to take out a neutron beam, pneumatic transport system, etc.

The VR-1 reactor facility provides education and training for students and professionals from Czech Republic and other countries. Training reactor has limited power, however its experimental instrumentation and well-equipped laboratories provides large experimental and educational capabilities. In addition to standard equipment (such as vertical and horizontal channels), the reactor 


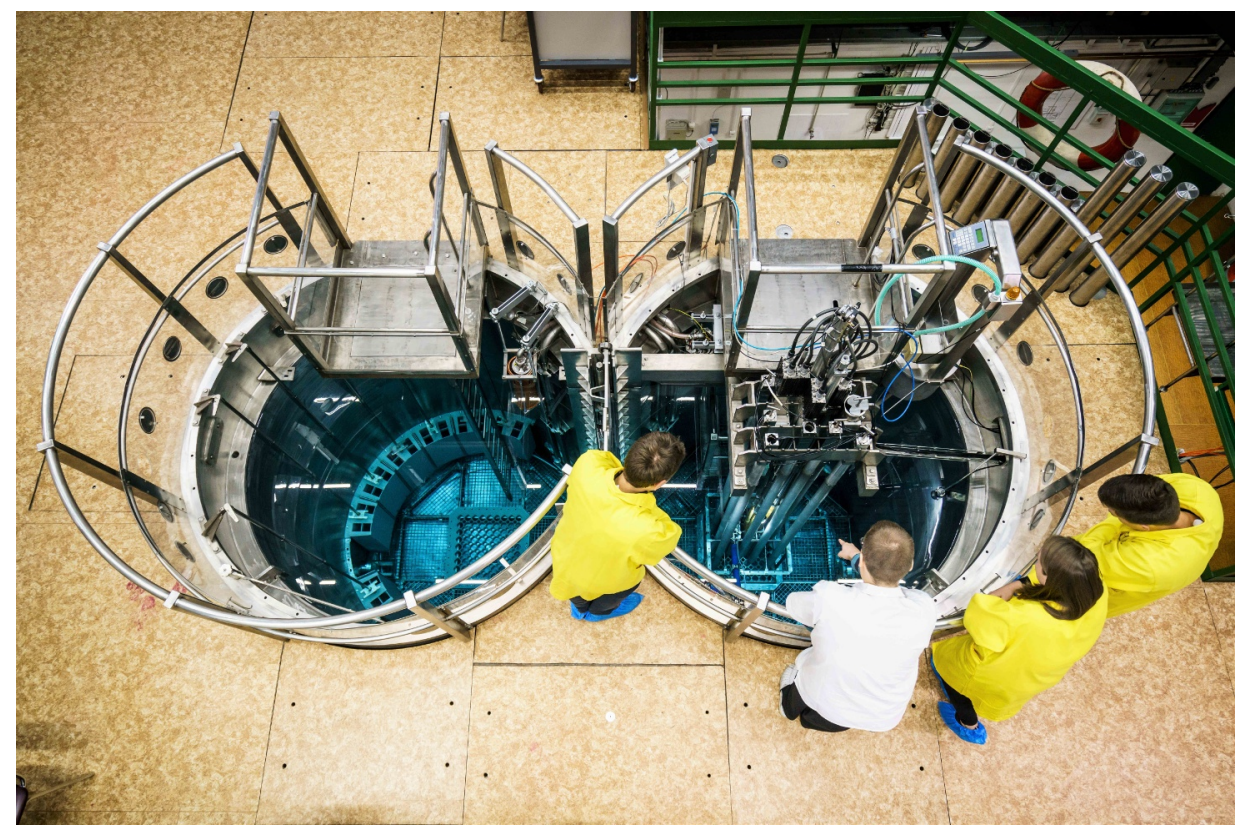

Figure 1: The Training reactor VR-1

is also equipped with several experimental devices especially developed for education and training such as a device for the detection of delayed neutrons; a device for the study of reactor kinetics; a device for the study of void coefficients and temperature coefficients. Facility contains four additional laboratories which are aimed at neutron activation analysis, radiation detection, neutron experiments, I\&C, reactor control and physical protection.

\section{Design of SCRAM Experimental instrumentation}

Experimental capabilities of the VR-1 reactor allows to study basic phenomena in the reactor core but do not combine basic principles study with reactor operation, and this reactor operation connection should be introduced in the new device. Later, the rod SCRAM instrumentation should be shared with other research reactors and after some modifications, the device will be possible to instal on other research nuclear reactors. Its design will take in account the similarity theory, so gained experience will be possible to transfer on other research reactors and large nuclear installations.

The device will consist of two major parts. The first part creates the transient process and the second part will be used for measurement of that process. In addition to this complex experimental device, methodology, data analysis software and models in reactor codes should be developed as well. This will allow to compare experimental data with results form calculation and than later improve code performance.

Conceptual design utilizes long experience with the VR-1 reactor operation, with experimental work and from the development of other reactor instrumentation. This experimental device may contain the control rod (with instrumentation, position measurement, drop delayer and drop break) 
and detection part (detectors and data acquisition system):

- the control rod with all instrumentation (motors, safety features) - so the fast transient process can be produced,

- fine measurement system for rod position (including the time synchronization) - so the exact position can be recorded during the experiment and later linked with data from detectors,

- the control rod drop delayer - device that can modify the speed of drop - so the reactor response on different insertion speed can be studied; Plus this may allow to study some accident conditions like overheated core with damaged guide tubes,

- the control rod break - so events, when the rod is inserted only partially, can be studied - due some troubles with guide tubes,

- inventory of different control rods (different design, materials - CdInAg, Boron,...),

- set of detectors in different positions (in vertical and horizontal locations) - so the flux changes can be measured, including the propagation of flux changes through the reactor core,

- electronic necessary for fast data acquisition (amplifiers, AD converters, $\mathrm{HV}, \ldots$ ).

\subsection{Diamond detector development}

Development of fast detection system is highly challenging task. Detectors should be small, fast and with high count rate detectability. In collaboration with colleagues from CEA and after first tests [3], the diamond detectors were selected as promising solution. Diamond detectors are developed for several application [4] [5] [6]: mainly in personal monitoring or accelerator beam measurement, but not for in-core reactor measurements. Therefore we started to modify diamond based detectors for thermal neutron measurements. This development take place mostly in CEA. Single crystal diamond with boron conversion layers were prepared and tested. Alpha particles from $(\mathrm{n}, \alpha)$ reaction interact within the diamond crystal and produce electron - hole. Deposited charge is collected by high voltage applied on crystal. Signal from detector is amplified by charge sensitive amplifier and the pulse shape is recorded. Pulse shape discrimination [7] is used for gamma - alpha separation. Several tests of the detector were performed with neutron sources at the VR-1 reactor and its laboratories. Preliminary results showed promising rates and yield. More detailed description of detection system development, detectors (Figure 2), pulse shape discrimination and results from our measurements are in [8] and in a paper that was submitted to impact journal and is currently under review.

\section{SCRAM analysis and calculation by Serpent code}

Proposed device will be equipped with detection system that allows to measure point flux development during transient. This rare potential of data generation should be combined with accurate and precise three-dimensional kinetic calculation. In order to, several codes were considered and reviewed. The Serpent code was selected and the modeling part was performed. 


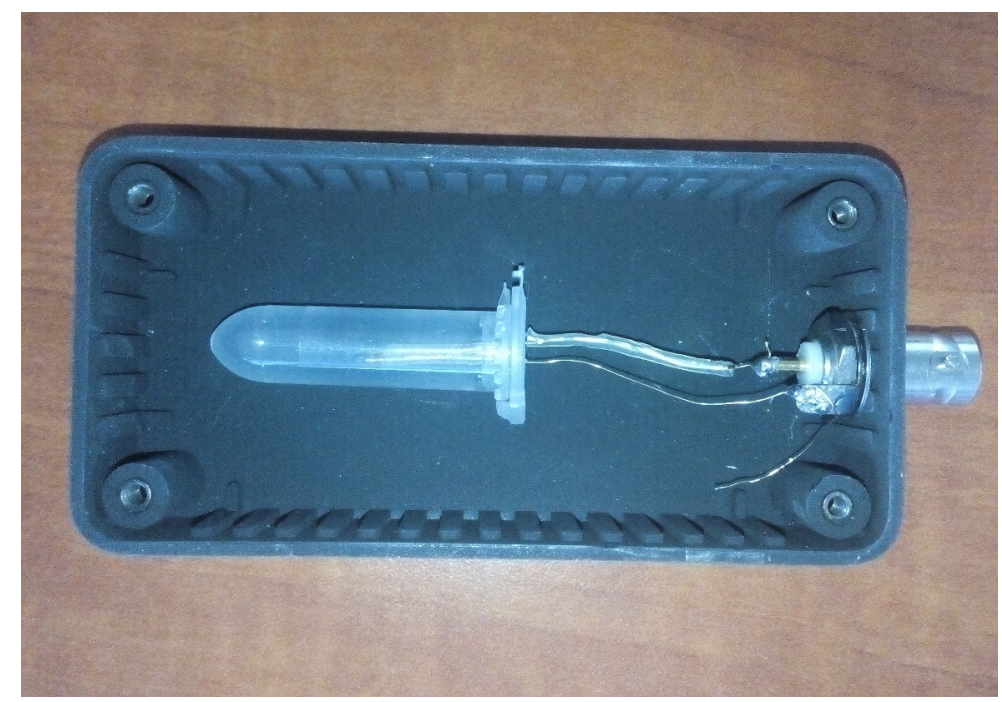

Figure 2: The diamond detector inside the plastic box

Serpent code contains dynamic toolkit, that allows to calculate reactor kinetics and dynamic using monte carlo approach. This unique tool is recently developed and is not widely used, however our first tests and first publication shows accurate results [9].

Details of the Serpent dynamic calculation are presented in [9], [10], [11], and [12], including the input syntax of time dependent model. Further details of the input syntax are described at the Serpent forum [13] and the Serpent wiki [14] (operated by Alto University). Serpent dynamic calculation is divided in two sub-calculations. This two step approach has a significant impact on the calculation accuracy [10]. The first calculation prepares a precursor distribution, i.e. the spatial density of delayed neutron precursors; the second calculates the transient itself.

A 3D core model of the VR-1 reactor was prepared for the first calculation. In the first calculation, neutrons and delayed neutron delayed precursors were tracked in the nominal model and recorded into a file. This file was later used in the second calculation, where changes in geometry and composition were introduced. In the case presented, the transient was initiated by rod drop. The duration of the drop was $0.5 \mathrm{~s}$.

\section{Preliminary results}

The development of the SCRAM experimental device is in the preparation phase. The threedimensional transient calculation was performed and obtained results can be considered as sufficient for future detail comparison (detail description is presented in a paper that was submitted to impact journal and is currently under review). The detection part is still under development (see [8] and in other paper that was submitted to impact journal and is currently under review), the problem with the data sampling and processing is currently under detail analysis.

The neutron flux development during and after rod insertion is shown in Figures 3. Data presented are from calculation by Serpent code, which used with JEFF3.3 nuclear library. Presented data 


\section{Table 1: Coefficients of linear fit for different cases with Asymptotic Standard Error and} the average value of control rod worth

\begin{tabular}{|c|c|c|c|}
\hline Source data & Slope $\left[\beta_{\text {eff }} / t\right]$ & Intercept $\left[\beta_{\text {eff }}\right]$ & Average \\
\hline Serpent full core & $0.010 \pm 0.009$ & $-1.92 \pm 0.02$ & $-1.908 \pm 0.005$ \\
Serpent detector zone 25 & $0.03 \pm 0.05$ & $-2.05 \pm 0.09$ & $-1.99 \pm 0.03$ \\
Serpent detector zone 13 & $0.08 \pm 0.03$ & $-2.20 \pm 0.06$ & $-2.06 \pm 0.02$ \\
Serpent detector zone 1 & $0.08 \pm 0.05$ & $-2.31 \pm 0.09$ & $2.18 \pm 0.02$ \\
Experiment & $-0.0012 \pm 0.0001$ & $-1.854 \pm 0.007$ & $-1.907 \pm 0.004$ \\
\hline
\end{tabular}

are obtained by full core neutron detector. The rod starts to drop at $0.1 \mathrm{~s}$ and is fully inserted at $0.63 \mathrm{~s}$. The shape of integral control rod worth results in neutron flux development with slow decrease followed by faster decrease of neutron population. After the rod full insertion, the neutron population decrease slowly. Calculation presented was focused at values after the insertion, which can be used for total control rod worth calculation.

The control rod worth can be used for comparison of the calculation and reactor experiment. This parameter can be obtained from the neutron flux development after the rod drop by inverse kinetic equation. The Table 1 presents control rod worth calculated by inverse kinetic with delayed neutron parameters from JEFF3.3 data library. Results are based on Serpent calculation and on data measured by LCM detector at the VR-1 reactor. The Serpent full core refers to data, that were obtained by calculation by detector, that contained whole reactor core. The Serpent detector zone $\mathrm{X}$ refers to detector placed in neighboring fuel cell to rod drop location. Number 1 marks position below reactor core, Number 13 position in the middle of the reactor core and number 25 refers to position above reactor core. Values of reactivity after drop were fitted by linear curve (see equation $1-a$ is Slope and $b$ is intercept) or average value was calculated.

$$
\rho=a \cdot t+b
$$

Values from the full core detector in Serpent calculation provided similar control rod worth as the experiment. The rod worth calculated by detector has slightly higher values, due the fact, that the control rod is inserted in neighboring fuel location. This results in more significant drop in the neutron flux. The axial difference in control rod value corresponds to the insertion direction - rod is inserted from top position.

\section{Conclusion}

Preliminary results of the rod drop experiment and calculation were presented to support the description of the new experimental device for the VR-1 reactor, which will be focused on dynamic experiments with the control rod. This device presented should allow to study the flux behavior during scram in several reactor core locations. The device described consist of two major parts. The first one is the control rod, the second part contains sets of detectors for neutron flux measurements. 


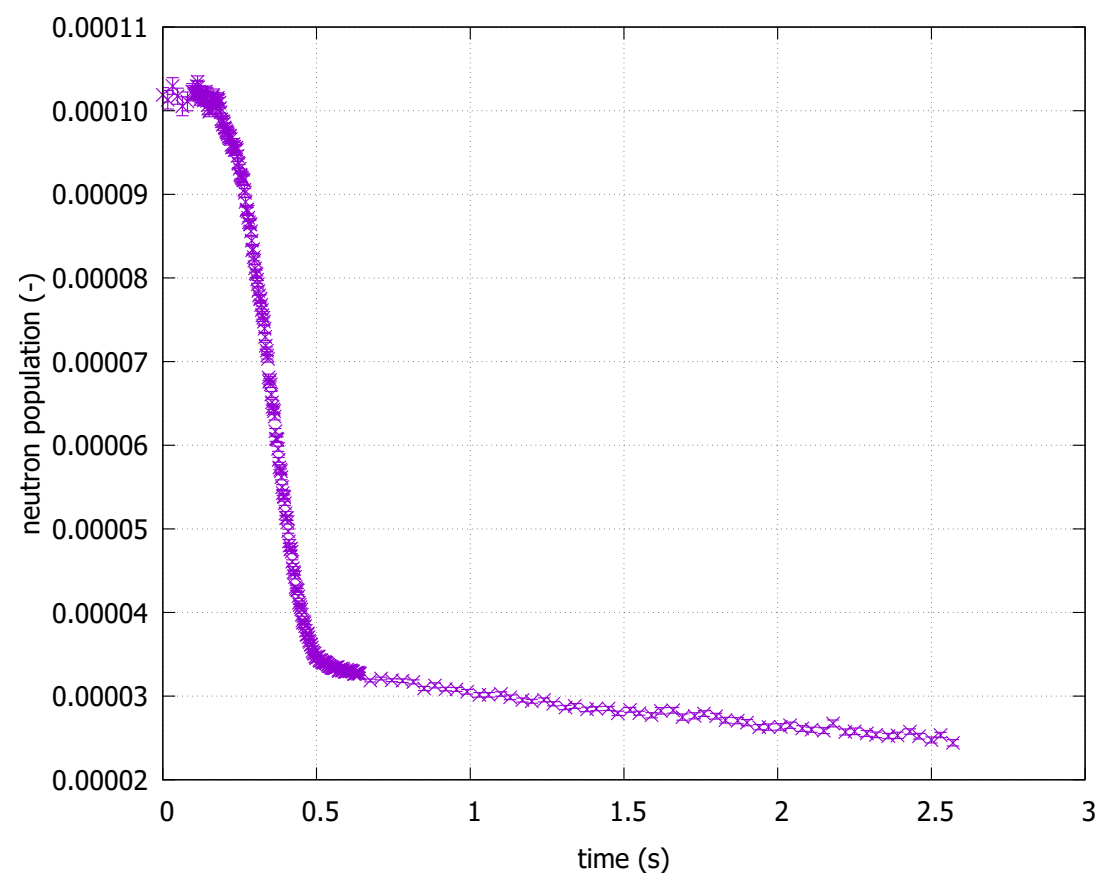

Figure 3: The neutron flux development during and after rod drop calculated by Serpent code

Results from Serpent calculation and VR-1 experiment are presented in Table 1. Serpent calculation is in agreement with experimental data especially for full core detector calculation. Rod worth calculated from Serpent detector in next to the rod is slightly higher, due the fact, that the control rod is inserted in neighboring fuel location. The axial difference in control rod value corresponds to the insertion direction - rod is inserted from top position.

\section{Acknowledgements}

This work was supported by several grants. The Fulbright Masaryk Grant from Fulbright Commission supported the research visit of The University of Tennessee which focused at transient calculation. Support from IAEA fellowship number C6/CZR/17003 was used for the research visit of CEA Saclay, where the diamond detectors were partially developed. The VR-1 reactor time was supported from the the project of Large research infrastructures by The Ministry of Education, Youth and Sports in Czech Republic - LM2015053 - VR-1 Training Reactor for Research Activities. The Grant Agency of the Czech Technical University in Prague, grant No. SGS18/146/OHK4/2T/14 supported work and material needs of the development at CTU.

\section{REFERENCES}

[1] L. Sklenka, J. Rataj, and F. Fejt. "Enhancing Experimental Capabilities of the Training Reactor VR-1 through Building VR-2 Subcritical Assembly and New Laboratories." In International Conference on Research Reactors: Addressing Challenges and Opportunities to 
Ensure Effectiveness and Sustainability. Buenos Aires, Argentina (2529 November 2019).

[2] J. Rataj. Reactor physics course at VR-1 reactor. Czech Technical University in Prague, Prague, 2 edition (2014).

[3] M. Pomorski. "Diamond as a solid state micro-fission chamber for thermal neutron detection." In Talks presented at the 3rd ADAMAS Collaboration Meeting, ECT*. Trento November 18 - November 20 (2014). URL http://www-adamas.gsi.de/ADAMAS03/talks/ Pomorski_CEA.pdf.

[4] R. S. Sussmann. CVD diamond for electronic devices and sensors, volume 26. John Wiley $\&$ Sons (2009).

[5] P. Bergonzo, A. Brambilla, D. Tromson, C. Mer, B. Guizard, R. Marshall, and F. Foulon. "CVD diamond for nuclear detection applications." Nuclear Instruments and Methods in Physics Research Section A: Accelerators, Spectrometers, Detectors and Associated Equipment, volume 476(3), pp. 694-700 (2002).

[6] G. F. Knoll. Radiation detection and measurement. John Wiley \& Sons (2010).

[7] M. Cerv. "CVD diamond application for particle detection and identification in high radiation environments." (2016).

[8] O. Novak, L. Sklenka, M. Pomorski, and C. Lynde. "Diamond detectors for fast transient SCRAM measurements - the detector development." Acta Polytechnica CTU Proceedings, $\mathrm{p}$. in print (2019).

[9] A. Levinsky, V. Valtavirta, F. P. Adams, and V. N. Anghel. "Modeling of the SPERT transients using Serpent 2 with time-dependent capabilities." Annals of Nuclear Energy, volume 125, pp. 80-98 (2019).

[10] J. Leppänen. "Development of a dynamic simulation mode in Serpent 2 Monte Carlo code." Proceedings of $M \& C$, pp. 5-9 (2013). URL http://www.fp7-hpmc.eu/papers/conference/M\& C-2013-7250.pdf.

[11] V. Valtavirta, M. Hessan, and J. Leppänen. "Delayed Neutron Emission Model for Time Dependent Simulations with the Serpent 2 Monte Carlo Code- First Results." Proceedings of PHYSOR, pp. 1-5 (2016). URL http://serpent.vtt.fi/mediawiki/images/b/b0/Valtavirta transients_PHYSOR2016.pdf.

[12] V. Valtavirta. "Coupled time dependent simulations with Serpent 2.1.27." Serpent UGM, Politecnico di Milano (2016). URL http://serpent.vtt.fi/mediawiki/images/3/3b/ ValtavirtaTransients.pdf.

[13] "Discussion forum for Serpent users." URL http://ttuki.vtt.fi/serpent/index.php.

[14] “Serpent Wiki.” URL http://serpent.vtt.fi/mediawiki/index.php/Main_Page. 